\title{
Vertically Stacked CMOS-compatible Photodiodes for Scanning Electron Microscopy
}

\author{
Lionel C. Gontard \\ Computer Sci. and Eng. Dept. \\ University of Cádiz (Spain) \\ lionel.cervera@uca.es
}

\author{
Juan A. Leñero-Bardallo \\ Inst. Microelectrónica Sevilla \\ (CSIC-Univ. of Seville, Spain) \\ juanle@imse-cnm.csic.es
}

\author{
Francisco M. Varela-Feria \\ Serv. Microscopía-CITIUS \\ University of Seville (Spain) \\ fmvarela@us.es
}

\author{
Ricardo Carmona-Galán \\ Inst. Microelectrónica Sevilla \\ (CSIC-Univ. of Seville, Spain) \\ rcarmona@imse-cnm.csic.es
}

\begin{abstract}
This paper reports the use of vertically stacked photodiodes as compact solid-state spectrometers for transmission scanning electron microscopy. SEM microscopes operate by illuminating the sample with accelerated electrons. They can have one or more solid-state sensors. In this work we have tested a set of stacked photodiodes fabricated in a standard 180nm HV-CMOS technology without process modifications. We have measured their sensitivity to electron irradiation in the energy range between $10 \mathrm{keV}$ and $30 \mathrm{keV}$. We have also assessed their radiation hardness. The experiments are compared with Monte Carlo simulations to investigate their spectral sensitivity.
\end{abstract}

Keywords—scanning electron microscopy, CMOS stacked diodes, high-energy electron detector

\section{INTRODUCTION}

Electron microscopy encompasses a set of techniques for characterizing materials from the signals generated by the impact of accelerated electrons. These measurements use to take the form of images. Electron microscopes (EM) are profusely used in science and industry. They allow studying the physical and chemical properties of materials and structures, with dimensions ranging from the atomic scale to hundreds of micrometers, e. g. semiconductor devices, catalysts, pharmaceutical products and biomolecules.

A fundamental capability of the EM is that it can be used for spectrometry, what provides additional information on the chemical composition of the materials. By interacting with atoms, electrons lose energy. This phenomenon is the source of a spectrometric technique called electron energy-loss spectrometry (EELS) [1]. The image sensors employed to detect these electrons only perceives intensity variations. The resulting images are grayscale, and therefore, electron spectrometry requires adding an extra device. This electron filter magnetically disperses electrons on a pixelated sensor on the basis of their energy, which is their color. However, this energy filter is a complex optical system consisting of magnetic lenses and electrostatic deflectors [2].

In this work, we explore a different approach. We aim to measure the energy of the electrons in a scanning electron microscope (Fig. 1(a)) operated in transmission mode, i. e. electron energy loss spectrometry (EELS), using a semiconductor sensor without the need of an electromagnetic energy filter [3][4]. The idea is to exploit the fact that electrons impinging in silicon generate free carriers at a certain depth form the semiconductor surface that depends on its energy (Fig. 1(d)). This phenomenon is similar to the case of light that is absorbed in silicon at a wavelength-dependent depth. This has been employed to design color sensitive pixels [5][6]. Particularly, we have investigated and compared the performance of photodiodes built with stacked p-n junctions operating in parallel versus conventional ones made with single p-n junctions [7]. We have characterized and compared the sensitivity of the photodiodes in the range of 10 to $25 \mathrm{kV}$ of accelerating potential. For this purpose, a dedicated chip has been fabricated in a standard 180nm HVCMOS technology. Four different sensor structures were implemented and compared. Experimental results are provided.

\section{SENSOR FOR ELECTRONS}

\section{A. Electron beam irradiation of silicon}

Scanning electron microscopes are the most extensively employed type of EM. They operate typically in the range of 1 to $30 \mathrm{keV}$ [8]. In these microscopes, the electron probe is focused on a point in the sample, and images are obtained by sequentially scanning the point-to-point probe and measuring the generated signals. A fundamental difference of electrons with respect to photons is that the interaction of an electron beam with a target material, e.g. silicon, results in a number of signals, such as secondary electrons (SE), Auger and backscattered (BSE) electrons, X-rays and emission of photons from luminescent materials. These SEM signals can be mapped point-by-point in two dimensions to build an image. The signals to form the images are captured using semiconductor based sensors typically formed by diodes. They can be direct- or indirectly coupled, and can be segmented into a discrete number of pixels with different geometries [9].
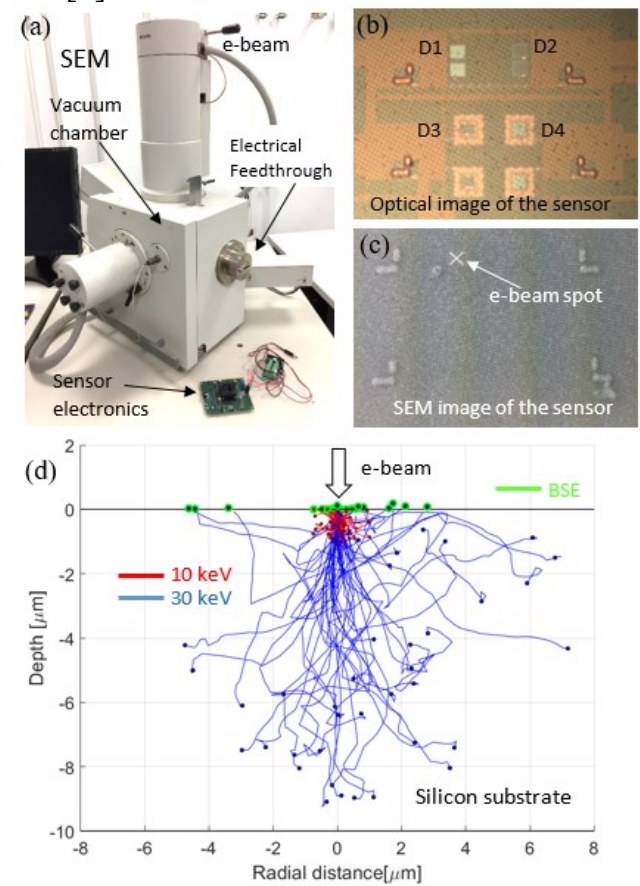

Fig. 1.(a) Scanning electron microscope. (b) Optical image of the sensor with 4 types of photodiodes. (c) SEM image of the sensor acquired with the SE detector. The boundaries of the diodes are not visible. The e-beam can be located at any position in the field-of-view. (d) Monte Carlo simulations of the trajectories and depth ranges of 50 electrons of $10 \mathrm{keV}$ or $30 \mathrm{keV}$ in silicon. Around $15 \%$ of the electrons are backscattered. 
When an accelerated electron impacts on a silicon diode, it loses energy and generates mobile charge carriers in the depletion region. The energy loss is gradual along the trajectory of the electron within the diode. A cloud of electron-hole $(\mathrm{e}-\mathrm{-}+\mathrm{h}+)$ pairs is generated in turn until the particle is completely absorbed or exits the sensor. The electron travel in the silicon bulk can span hundreds of micrometers within the so-called interaction volume. If this volume is approximated by a sphere, its diameter $R$, i. e. the maximum travelled distance, can be estimated using one of the several semi-empirical expressions presented in the literature, for instance by Kanaya-Okayama [9],

$$
R_{K-O}=\frac{0.0276 A}{\rho Z^{0.89}} E^{1.67} \quad[\mu \mathrm{m}]
$$

or by Potts [11],

$$
R_{P}=\frac{0.1}{\rho} E^{-1.5} \quad[\mu \mathrm{m}]
$$

In these equations the variable $A$ is the atomic weight $[\mathrm{g} / \mathrm{mol}], E$ is the energy of the incident beam $[\mathrm{keV}], \rho$ is the density of the material $[\mathrm{g} / \mathrm{cm} 3]$ and $Z$ is the atomic number. Fig. 2.(a) plots the variation of $R_{K-O}$ and $R_{P}$ as a function of incident energy for silicon using the parameters for silicon: $A$ $=28.085 \mathrm{~g} / \mathrm{mol}, Z=14, \rho=2.23 \mathrm{~g} / \mathrm{cm} 3$, and $E$ take values in the range of $10-30 \mathrm{keV}$ in steps of $5 \mathrm{keV}$. The curves show that the interaction volume of the incident electron increases with its kinetic energy, spanning from $1 \mu \mathrm{m}$ at low incident energies to several $\mu \mathrm{m}$ at $30 \mathrm{keV}$. These distances are compatible with the depth of the junctions of the different stacked photodiodes available in standard CMOS integration technologies (see Fig. 2.(b)). Moreover, compared to nonstacked conventional structures, stacked ones have the potential for increased sensitivity and spectral selectivity. This latter feature refers to detection of photons of different frequency by using junctions placed at different depths within the semiconductor, exploiting the fact that absorption depths depend on photon wavelengths [7]. Our target is to compare the sensitivity and spectral selectivity of several stacked diodes for evaluating their potential application in sensors for electrons in the range of energies used in SEM.

\section{B. Standard stacked photodiodes}

Fig. 1(b) shows a microphotograph of the chip whose dimensions are $1050 \mu \mathrm{m} \times 850 \mu \mathrm{m}$. The fabrication of the diodes and associated circuitry is described in [9]. The chip contains 4 types of photodiodes fabricated with standard $180 \mathrm{~nm}$ HV-CMOS technology. We have implemented two diodes of each type D1-D4 (see Fig. 1(b)) connected in parallel to one integrate-and-fire (I\&F) circuit, depicted in Fig. 2. (c). They have exactly the same sizes $(16 \mu \mathrm{m} \times 13 \mu \mathrm{m})$. Their depths and doping strengths were not disclosed by the foundry, thus the depth and width of the depletion regions is not known with any accuracy. We have estimated that the thicknesses are $0.2 \mu \mathrm{m}, 0.8 \mu \mathrm{m}$ and $1 \mu \mathrm{m}$ for the upper, middle and lower diffusions respectively, larger than the ones reported in for standard 90nm CMOS technology [13]. Photodiodes D1 and D2 are built by stacking 2 and 3 different diodes involving deep n-well and deep p-well layers. The other two D3 and D4 are classic photodiodes available in standard CMOS technologies, i.e. diodes either made with nwell/p-substrate junction or with a $n$-diffusion/p-substrate junction.
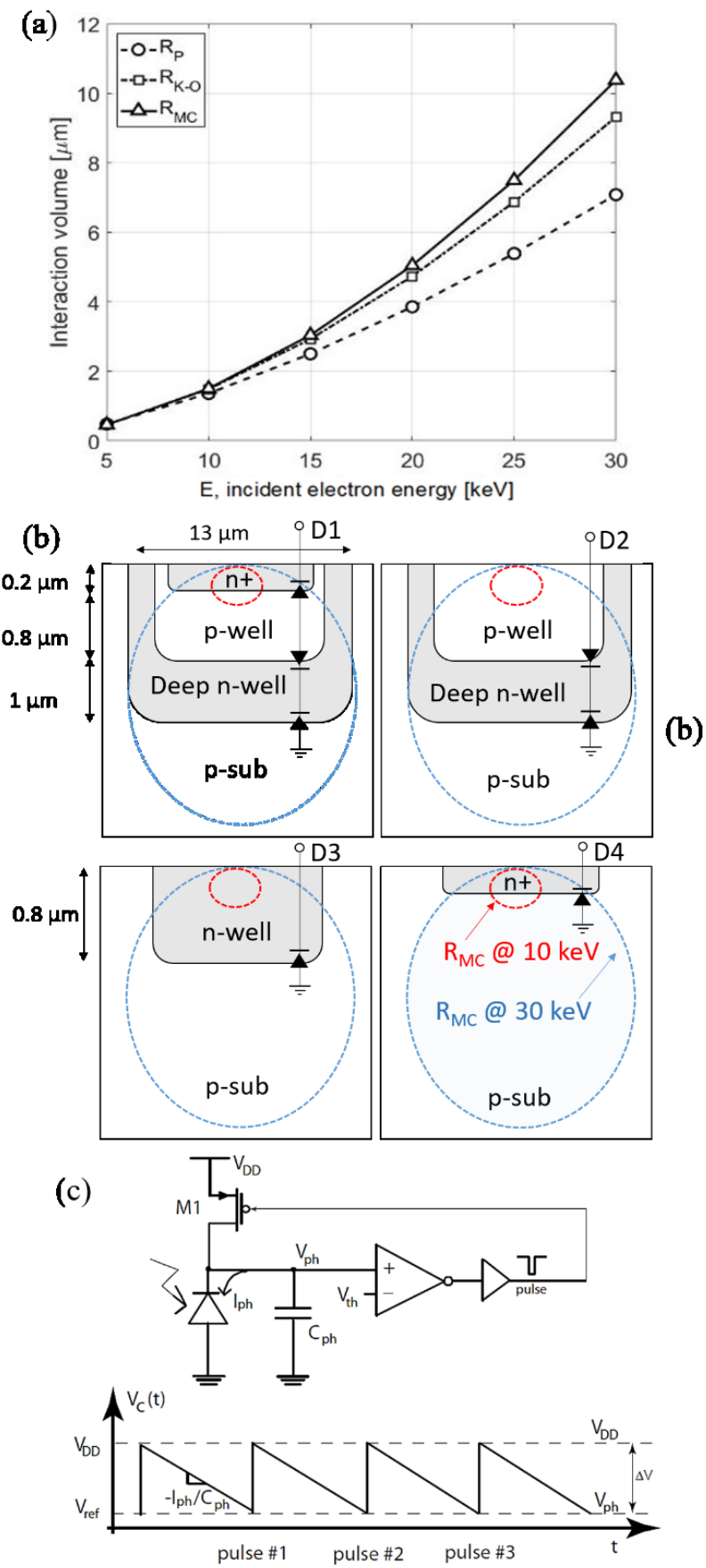

Fig. 2.(a) Depth ranges of electrons in silicon as a function of energy calculated using analytical and Monte Carlo simulations. (b) The four types of diodes studied in this work. Dashed lines indicates the depth range of electrons bombarded onto the diodes with $10 \mathrm{kev}$ (red dashed line) and $30 \mathrm{keV}$ (blue dashed line). (c) Astable oscillator implemented for each diode.

Taking into account the diodes dimensions and their depth, different spectral sensitivity is expected for them [7]. The upper ones are more sensitive to blue light and the bottom ones have sensitivity peaks in within the Near Infrared (NIR) bands. Diodes D1 and D2 are compounded of two and three diodes operating in parallel respectively. They provides higher current than the single ones and have a more uniform response in the visible and the NIR bands. Diodes D3, and 
D4 are single diodes with sensitivity peaks in different spectral regions. Knowing their spectral response, it is possible to infer the response of the middle diode in the diode configurations D1 and D2.

Each set of photodiodes is connected to an astable oscillator that switches between two states at the pace defined by the input photocurrent. With this simple circuitry [7], photodiodes photocurrents can be measured and compared just counting pulses during a time interval, avoiding the use of complex equipment to gauge currents inside the microscope. The circuit is described in Fig. 2.(c). Hence, if the diodes are irradiated with a constant electron beam current, the measured oscillation frequency should be proportional to the electron beam energy.
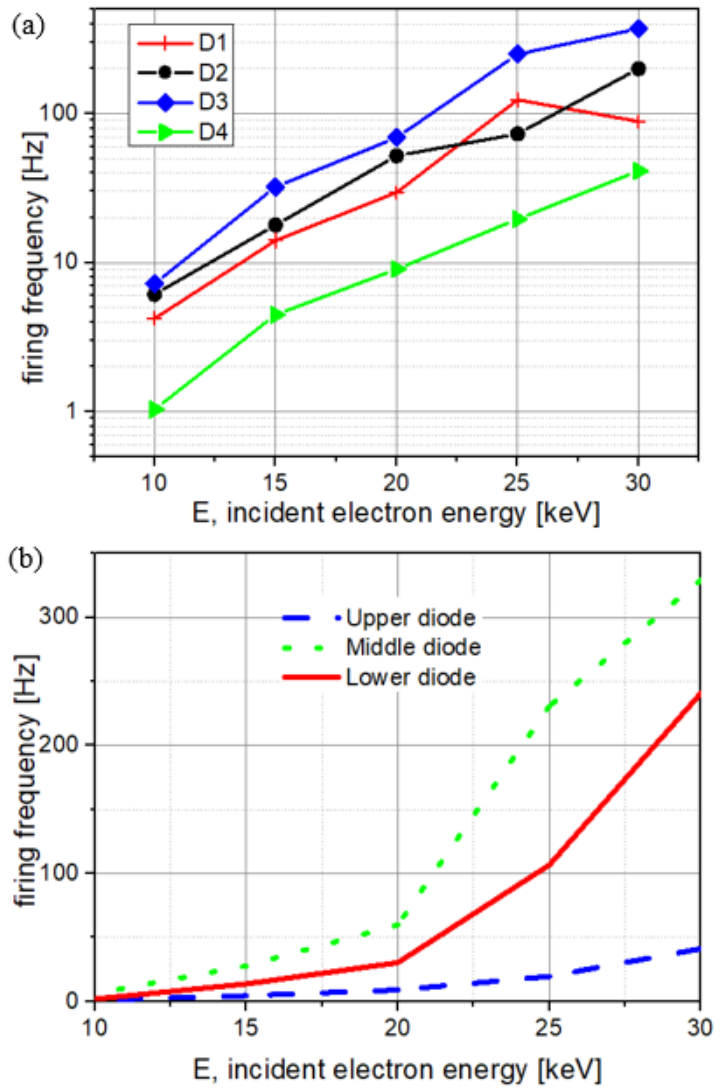

Fig. 3.(a) Spiking frequencies versus electron beam energy for a fixed beam current for the diodes D1 to D4 with logarithmic scale (b) Effective charge in terms of the firing frequency in each diode at different depths with linear scale.

\section{EXPERIMENTS}

\section{A. Experimental setup}

The sensor chip with the four stacked photodiodes was mounted on a PCB with power regulation and connectors to read out the spiking frequency of each diode. The PCB was located inside the vacuum chamber of a SEM FEI XL 30. The SEM was equipped with a secondary electron detector that could be used to image the chip and to place the electron beam on top of each of the 4 photodiodes. During the experiments, all the photodiodes were exposed to an electron beam current of the same magnitude, namely $1 \mu \mathrm{A}$, by fixing the aperture, and a probe size of $3.5 \mathrm{~nm}$ (spot size $6.7 \mathrm{~mm}$ and working distance $51 \mathrm{~mm}$ ).

\section{B. Comparison of the spectral sensitivity of the diodes}

Fig. 3.(a) plots the firing frequencies of the four diodes D1-D4 as a function of the incident electron energy. The most sensitive is D3 and D4 the less one.

The curves in Fig. 3.(b) have been calculated from the curves in Fig. 3.(a) using the following arithmetic relations: Upper $=$ D4, Middle $=|\mathrm{D} 3-\mathrm{D} 4|$ and Lower $=|\mathrm{D} 3-\mathrm{D} 4-\mathrm{D} 1|$, corresponding to the diffusion of the Upper, Middle and Lower diodes respectively. The curves show that spectral selectivity is plausible using the signal generated by stacked photodiodes.

\section{ELECTRON INTERACTION CALCULATIONS}

\section{A. Monte Carlo simulation of spectral sensitivities}

In order to accurately evaluate the spectral sensitivity of the diodes we have carried out some computer simulations. The cloud of carriers generated by the collision of electrons within the silicon sensor were calculated using Monte Carlo simulations. In particular we applied the plural scattering model, which is accurate and fast for bulk samples [13][14]. Compared with the more exact single scattering model, the plural scattering model makes some assumptions like that every electron travel exactly the same total path length within the specimen before coming to rest. That distance is found by numerically evaluating the integral:

$$
R_{B}=\int_{0}^{E}\left[\frac{-1}{\frac{d E}{d S}}\right] d E
$$

which computes the total distance measured along trajectory $S$ that is required for an electron starting with energy $E$ to give up all of its energy. Here $d E / d S$ is the stopping power given by the modified Bethe equation suggested by Joy and Luo in 1989 given as

$$
\frac{d E}{d S}=-78500 \frac{Z}{A E} \ln \left(\frac{1.166(E+0.85 J)}{J}\right)
$$

where $J$, measured in $\mathrm{keV}$, is known as the mean ionization potential and represents the effective average energy loss per interaction between the incident electron and the solid. $J$ has been measured experimentally for a wide range of compounds, and for silicon is approximately $0.173 \mathrm{keV}$.

\section{B. Monte Carlo simulation of electron-induced free charge}

We use simulations for computing the fraction of electrons that are backscattered by the sample, which are those electrons that are elastically scattered out of the surface, and hence do not generated free carriers. The integral in Eq. (4) is calculated using a fixed number of discrete steps so that the energy of the electron after each step is given by

$$
\Delta E=\int_{0}^{E}\left[\frac{d E}{d S}\right] d S
$$

The electron trajectory is erratic within the sample, so that $\Delta E$ is a function of the radial distance and the depth. The energy required to create a electron-hole pair in silicon is $3.6 \mathrm{eV}$, and the equation can be integrated to calculate the number $n$ of free carriers generated in a cylindrical region of 
the silicon sensor of radius $r 1$ and thickness $\left|z_{1}-z_{2}\right|$ using the equation:

$$
n=\int_{0}^{r 1} \int_{z 1}^{z 2} \frac{\Delta E}{3.6} d r d z \quad\left[e^{-}-h^{+} \text {pairs }\right]
$$

We have employed Eq. (6) to calculate the free charge that is generated and presumably collected by each of the Upper, Middle and Lower diffusions. This is obtained by integrating in the radial direction $(d r)$ between 0 and $\infty$ and integrating in the depth dimension $(d z)$ in three depth ranges defined in Table I.

Table I. Effective depth ranges of stacked diodes

\begin{tabular}{cccc}
\hline Depth limits $(\mu \mathrm{m})$ & Upper & Middle & Lower \\
\hline$z 1$ & 0 & 0.31 & 1.71 \\
$z 2$ & 0.3 & 1.7 & 2.3 \\
\hline
\end{tabular}

Fig. 4(a) shows the results of the simulation of the free charge generated by one incident electron of energy $E$ in each of the three stacked diffusions. The calculations assume that the number of electrons (beam current) that impacts the sensor is the same at all energies.
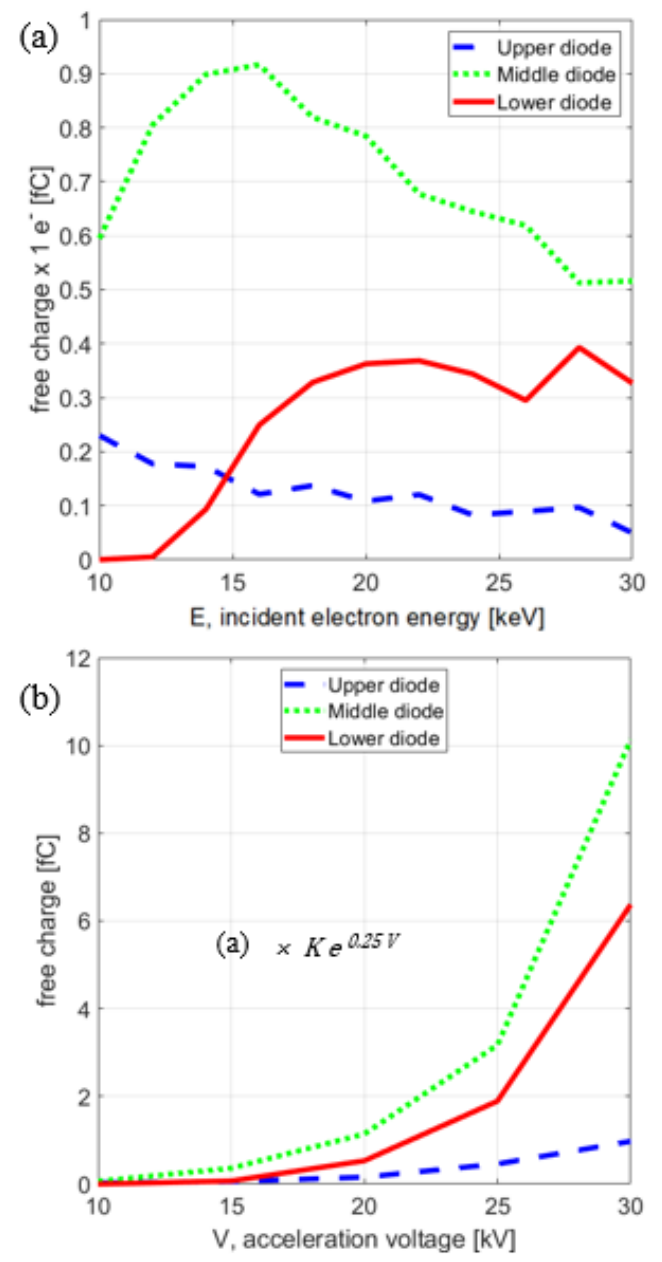

Fig. 4.(a) Free charge generated in each diffusion calculated using Monte Carlo simulations. (b) Free charge generated in each of the three stacked diodes adding the influence of the I-V characteristics of the electron beam of the SEM.
The shapes of the curves in Fig. 4(a) cannot be compared with the experimental curves of Fig. 3. (b). This is because in our experiments the beam current cannot be fixed. In fact, the acceleration voltage in a SEM also affects the beam current. Typically, the beam current grows exponentially with the acceleration voltage. Fig. 4(b) show the curves of Fig. 4(a) after multiplication by the electron beam current-acceleration voltage (I-V characteristic of the SEM) which has the form $I=K e^{b V}$. As the data of the characterization of parameters $K$ and $b$ are not known, $K$ was set to an arbitrary value, and we found that the shapes of the simulated curves, and their ratios could be fitted qualitatively to the experimental ones using a value of $b=0.25$.

\section{CONCLUSIONS}

We have tested a set of stacked photodiodes irradiated with the electron beam produced in a SEM for their potential use as spectral detectors for transmitted electrons (EELS). Experimental measurements display good spectral sensitivity within the $10-30 \mathrm{keV}$ spectral region. The experiments have been accurately modelled using Monte Carlo simulations and the plural scattering approximation. Our experiments also confirmed that the diodes could stand safely electron irradiation without apparent functional damage. In terms of energy resolution, the solid-state detector described here cannot compete with electromagnetic filters. However, the advantages of this type of pixel structure are the use of a cheap and standard 180nm HV-CMOS technology, not requiring additional devices like electromagnetic prisms but with an energy resolution comparable to other detectors used for energy-dispersive x-ray spectrometry, and the possibility to integrate the sensor with other CMOS circuitry on a single die.

\section{ACKNOWLEDGMENTS}

The authors want to acknowledge funding from MCIU/AEI/ERDF-EU through projects PGC2018-101538A-I00 and RTI2018-097088-B-C31. Financial support from the program ASECTI and Plan Propio-UCA Ref. 18INPPPR05 are also acknowledged. Also from EU H2020 MSCA through Project ACHIEVE-ITN (Grant No 765866) and by Ayudas a Proyectos de I+D+I del Programa Operativo FEDER through Project US-1264940.

\section{REFERENCES}

[1] R. F. Egerton, "Electron energy-loss spectroscopy in the TEM", Reports on Progress in Physics, vol. 72, no. 1, 2008.

[2] H. H. Rose, "Optics of high-performance electron microscopes," Science and Technology of Advanced Materials, vol. 9, no. 1, 2008.

[3] T. Klein, E. Buhr, and C. G. Frase, "TSEM: A review of scanning electron microscopy in transmission mode and its applications," in Advances in imaging and electron physics, vol. 171, pp. 297-356). Elsevier, 2012.

[4] T. Luo and A. Khursheed. "Transmission EELS Attachment for SEM," IEEE Transactions on device and materials reliability, vol. 6, no. 2, June 2006.

[5] S. M. Sze, "Semiconductor Devices Physics and Technology". Hoboken, NJ, USA: Wiley, 2006.

[6] Foveon Inc. (Feb. 2016). [Online]. Available: http://www.foveon.com

[7] Juan Antonio Leñero-Bardallo, Manuel Delgado-Restituto, Ricardo Carmona-Galán, and Ángel Rodríguez-Vázquez "Enhanced sensitivity of CMOS image sensors by stacked diodes". IEEE Sensors Journal, Vol. 16, no. 23, December 1, 2016. 
[8] J. I. Goldstein, D. E. Newbury, J. R. Michael, N. W. Ritchie, J. H. J. Scott, and D. C. Joy, "Scanning electron microscopy and X-ray microanalysis". Springer, 2017.

[9] L C. Gontard, Grigore Moldovan, Ricardo Carmona-Galán, Chao Lin, and Angus I. Kirkland. "Detecting single-electron events in TEM usinglow-cost electronics and a silicon strip sensor". Microscopy, , pp. 119-130, 2014

[10] K. A. Kanaya and S. Okayama, "Penetration and energy-loss theory of electrons in solid targets," Journal of Physics D: Applied Physics, vol. 5, no. 1, p. 43, 1972.

[11] P.J. Potts, "A Handbook of Silicate Rock Analysis", Springer, Dordrecht 1987.

[12] A. Polzer, K. Schneider-Hornsteina, J. Donga, P. Kostova, and H. Zimmermann, "Investigation of triple-junction photodetector in $90 \mathrm{~nm}$ CMOS technology," in 2011 Proc. Eurosensors XXV, Sept 2011, Athens, Greece.

[13] L. Curgenven and P. Duncumb. "Simulation of electron trajectories in a solid target by a simple Monte Carlo technique. Report 303," in Tube Investments Res. Lab., Saffron Walden, Essex, 1971.

[14] Joy, D. C.. Monte Carlo modeling for electron microscopy and microanalysis, vol. 9. Oxford University Press, 1995. 